\title{
Online Peer Assessment: pontos e contrapontos de docentes e de estudantes
}

\author{
Selma Santos Rosa, Camila A. Macedo, José E. S. Geremias Jr., Eliana S. \\ Lisboa, Rodrigo C.T. Souza, Valdir Rosa \\ Universidade Federal do Paraná \\ Paraná, Brasil
}

\{selmasantos, camila.macedo, jose.junior943, eliana.lisboa,thom,valdirrosa\}@ufpr.br

\begin{abstract}
This article reports on findings from a study aimed at analyzing the ways in which Online Peer Assessment (OPA) has been developed in higher education taking into account both teachers' and students' perceptions. Assessment methods and digital technologies used in OPA were identified. Also, the students' point of view were analyzed regarding OPA practices developed by teachers and related to existing literature. As a result, suggestions to develop OPA and to implement innovative pedagogical proposals mediated by Digital Technologies are presented in order to promote students' autonomy, the production of constructive feedback and the development of critical thinking.
\end{abstract}

Resumo. Com a proposição de compreender, a partir de práticas de docentes e de estudantes, como a Online Peer Assessment (OPA) tem sido desenvolvida no ensino superior, inquerimos docentes e estudantes; identificamos diretivas de avaliação e Tecnologias Digitais utilizados em OPA; relacionamos pontos de vista de estudantes com práticas de OPA desenvolvidas pelos docentes e as confrontamos com a literatura. Como resultado, apresentamos indicações que podem intensificar o desenvolvimento de OPA e a concretização de propostas pedagógicas inovadoras mediadas por Tecnologias Digitais, com vista à promoção da autonomia do estudante, da produção de feedback construtivos e do desenvolvimento do pensamento crítico.

\section{Introdução}

Historicamente, a ênfase dos processos de avaliação tem-se manifestado como medidas de classificação (avaliação somativa), com pouca ênfase na avaliação como uma via para contribuir com o aprendizado dos alunos (avaliação formativa). Caseiro e Gebran (2008, p.2) enfatizam que a avaliação "tem servido quase que exclusivamente como instrumento de verificação, seleção e classificação. Quase nenhuma atitude no sentido de reorientar a prática educativa é tomada diante dos dados coletados pela avaliação." Por outro lado a aprendizagem ao longo da vida e o desenvolvimento de habilidades nas pessoas que as conduzam ao pensar criticamente e a capacidade de avaliar a qualidade do seu próprio processo e resultados de aprendizagem, tanto durante quanto após a sua formação, são temas majoritariamente incentivados. 
VII Congresso Brasileiro de Informática na Educação (CBIE 2018)

Anais do XXIV Workshop de Informática na Escola (WIE 2018)

De acordo com o Assessment Reform Group [ARG 1999], grupo de estudos de reformas da avaliação da aprendizagem, a avaliação é uma das ferramentas educacionais mais poderosas para promover aprendizagens eficazes, se utilizada da maneira correta. Dos estudos da ARG surgiu o termo "Avaliação para a Aprendizagem" (ApA), definido como um processo utilizado por professores e alunos na busca e na interpretação de evidências da aprendizagem. Ao reconhecer que os únicos responsáveis por seu aprendizado são os próprios aprendizes, pois ninguém pode fazer isso por eles, a ApA envolve-os de modo a dar-lhes informações sobre seu desempenho e orientação sobre seus esforços seguintes. Essa informação é fornecida, principalmente através do feedback do professor, de seus pares e, também, através do seu envolvimento ativo nos processos de avaliação, implementando práticas formativas de avaliação para apoiá-lo em suas trajetórias de aprendizagem.

Dentre os métodos de ApA, destacamos neste artigo a Online Peer Assessment (OPA), desenvolvida proeminentemente por meio de tecnologias online. Com ela enfatiza-se o envolvimento do estudante no processo de sua própria avaliação e na dos seus pares e isto é cognitivamente mais exigente e permite envolvê-los de forma mais ativa na aprendizagem e no exercício do pensamento crítico, habilidades essenciais para a vida profissional contemporânea, onde os estudantes (futuros profissionais) terão que, constantemente, definir metas e avaliar por conta própria seus objetivos, suas estratégias e suas decisões [Nicol 2011]. Dar aos estudantes a oportunidade de envolvimento nos processos de avaliação, implica fazê-los entender os critérios de avaliação utilizados e, por isso, é fundamental incluí-los na concepção das avaliações e atribuir a eles mais responsabilidade nesse processo e, consequentemente, na sua aprendizagem [Santos Rosa, Coutinho e Flores 2017].

No presente artigo, temos a proposição de contribuir com pesquisas sobre avaliação pelos pares mediadas por Tecnologias Digitais online. Para isso identificamos Tecnologias Digitais utilizadas por docentes em OPA; relacionamos pontos de vista de estudantes com práticas de OPA desenvolvidas por docentes e; analisamos as considerações de docentes e de estudantes acerca da OPA no ensino superior a partir de dados empíricos e de pesquisas afins. Como resultado, buscamos intensificar o desenvolvimento da autonomia, da produção de feedback construtivos e do pensamento crítico do estudante, concretizando propostas pedagógicas mais ousadas e inovadoras do ponto de vista da ApA, mediada por Tecnologias Digitais online.

\section{Procedimentos metodológicos}

Compartilhamos parte de uma pesquisa realizada no âmbito de um projeto de cooperação internacional entre a Universidade do Minho e a Universidade [Santos Rosa, Coutinho e Flores 2017], cuja proposição é a pesquisa educacional e o desenvolvimento de Tecnologias Digitais para ApA. Para isso seguiremos um modelo de estudos mistos, com uma primeira fase de natureza com predomínio qualitativo em que foram feitas entrevistas com 4 docentes de três universidades portuguesas (Universidade Trás-osMontes e Alto Douro, Universidade do Minho e Universidade de Aveiro). A definição desses docentes deu-se a partir da adesão dos mesmos para contribuir, tendo em vista que desenvolvem OPA. Já na segunda fase da pesquisa de cariz quali-quantitativo, realizamos um inquérito por questionário online disponibilizado no google forms, o qual foi respondido por 50 estudantes que participam de OPA ao longo do Mestrado Integrado em Engenharia e Gestão Industrial da Universidade do Minho. 
VII Congresso Brasileiro de Informática na Educação (CBIE 2018)

Anais do XXIV Workshop de Informática na Escola (WIE 2018)

A entrevista com os docentes (identificados na seção 3 como: prof1, prof2, prof3 e prof4), obteve um formato oral semiestruturado e foi organizada em um guia composto por 19 questões abertas. Já o questionário, adaptado de Wen e Tsai (2006), foi submetido aos estudantes com 20 questões fechadas organizadas em uma escala Likert, sendo:01 (discordo totalmente) a 05 (concordo totalmente), complementadas com 04 questões abertas.

A partir de uma Revisão Sistemática da Literatura sobre peer assessment no ensino superior, realizada em uma pesquisa anterior [Santos Rosa, Couitnho e Flores 2017], correlacionamos os dados empíricos obtidos com os docentes e com os estudantes, organizados em duas categorias de análise: (1) Ponto de vista de docentes e de estudantes sobre práticas de OPA. Identificamos pontos frágeis e fortes relacionadas a OPA, a partir das experiências vividas pelos participantes desta pesquisa (docentes e estudantes); (2) Tecnologias Digitais utilizadas em contextos de OPA na fase de desenvolvimento (que ocorre quando o estudante-avaliado está envolvido no processo de implementação da atividade e utiliza uma ou mais dessas tecnologias para gerar um produto) e na fase de avaliação dos resultados, referente ao engajamento dos estudantes nas tarefas e no processo de avaliação pelos pares. Esta fase se estabelece da interação entre os pares e é realizada tanto durante quanto após a fase de desenvolvimento de OPA.

Passamos a apresentação dos resultados da intervenção com os docentes e com os estudantes.

\section{Resultados e Discussão}

De modo geral, quando se inicia a implementação de uma OPA, surgem diversas questões tanto por parte dos estudantes quanto por parte dos docentes. Dentre elas, as mais comuns são: Quem recebe nota da(o) docente: o par-avaliador ou o par-avaliado? Ou ambos, ou ainda nenhum deles? A nota atribuída pelo par-avaliador ao par-avaliado é contabilizada na média final do par-avaliado? Ao atribuir uma nota ao par-avaliado perde-se, por questões de exposição de ideias contrárias ou outros fatores, a qualidade na produção do feedback elaborado pelo par-avaliador? Como ocorre a organização das atribuições de notas quando os alunos são organizados em grupos? Um grupo atribui uma única nota a outro grupo? Cada membro de um grupo avalia o trabalho do outro grupo? Quais são os tipos de resultados ou produtos avaliados em OPA: um texto, um software, outros? A nota da avaliação pelos pares implica na nota final do par-avaliado? A OPA deve garantir o anonimato dos estudantes? Na OPA há um grande número de registros gerados a partir das avaliações feitas entre os alunos e isso conduz a inviabilidade de praticá-la em turmas com grande quantidade de alunos? A OPA prevê situações de injustiças? Como evitá-las? Existem softwares específicos para OPA?

A seguir sintetizaremos respostas a estas perguntas, a partir das pontos de vista de docentes e de estudantes, conforme expusemos na seção 2.

\subsection{Ponto de vista de docentes e de estudantes sobre práticas de OPA}

As avaliações pelos pares remetem para a discussão de um paradigma que enfatiza a centralidade do estudante e os objetivos de formação, colocando o enfoque na aprendizagem e no seu papel ativo, o que remete para funções diferenciadas para o estudante e para o docente com implicações nos procedimentos da avaliação 
VII Congresso Brasileiro de Informática na Educação (CBIE 2018)

Anais do XXIV Workshop de Informática na Escola (WIE 2018)

[Bouchoucha e Wozniak 2010; Grez e Valcke 2013; Chang et al. 2012; Eugenia 2014; Grez e Valcke 2013; Al-Smadi, Guetl e Kappe 2009].

Sluijsmans, Straetmans e Merriënboer (2008) consideram que a avaliação é normalmente o elemento de maior importância do ponto de vista do estudante. E por isso, é fundamental abordar a concepção das avaliações e atribuir a eles mais responsabilidade nesse processo e na sua aprendizagem, sendo que, para isso, é necessário progredir em métodos e recursos tecnológicos que sustentem tal consideração.

Os docentes entrevistados utilizam procedimentos de avaliação diversificadas. Prof1 avalia o desempenho do grupo-avaliador e do grupo-avaliado. Os resultados alcançados nessas avaliações, representam uma estratégia do processo de ensinoaprendizagem com vistas a construção do conhecimento do estudante no contexto da aprendizagem colaborativa. Esta docente considera que quando a avaliação de desempenho é atribuída pelo par-avaliador, sendo que este deve associar uma classificação juntamente com um comentário, esta classificação pode desvirtuar o efeito do feedback.

Nas disciplinas de prof4, as OPA são realizadas nos $1^{\circ}, 8^{\circ}$ e $9^{\circ}$ semestres do curso. Nos $1^{\circ}$ e $8^{\circ}$ semestres cada grupo recebe uma nota dada pelo docente e uma nota dada por seus pares, sendo que, esta última conduz a notas diferentes entre indivíduos do mesmo grupo, a qual dependerá do desempenho individual nas tarefas de acordo com as pontos de vista dos colegas dos grupos. Já no $9^{\circ}$ semestre, cada estudante faz de 5 a 10 tarefas, as quais são submetidas a avaliações individuais, e também uma avaliação do trabalho do grupo, ambas feitas pelo docente. Nas avaliações pelos pares desenvolvidas no $8^{\circ}$ e $9^{\circ}$ semestres, são desenvolvidas estratégias que envolvem os estudantes em ambientes reais de trabalhos profissionais, onde eles são submetidos a tarefas de desenvolvimento de um projeto requerido por uma empresa real. Para isso, são criados dois segmentos: cliente e fornecedor. São definidos papeis e tarefas para assumirem ao longo desses dois semestres (por decisão/preferência dos próprios estudantes). Seis estudantes assumem o papel de clientes e os demais distribuem-se em grupos compostos por 6 estudantes cada, para assumirem os papeis e as tarefas de fornecedores de serviços ou produtos demandados pelos clientes (as turmas são compostas de em média 40 estudantes). No final, somente os 6 clientes apresentam o projeto à empresa requisitante. Os estudantes clientes recebem avaliação do docente e da empresa. Já os fornecedores, são avaliados em suas tarefas pelo docente e pelos estudantes clientes.

Os resultados produzidos nas OPA do prof4, variam de acordo com o projeto, como por exemplo: um relatório escrito e um protótipo físico feito em Lego mindstorms. Para finalizar, este docente enfatiza a importância de os estudantes perceberem que a avaliação pelos pares influencia nas notas deles. E, por isso, considera importante fazer com que a nota dessa avaliação implique na nota final do estudante.

Já a prof2, avalia o desempenho tanto do par-avaliado quanto do par-avaliador. Entretanto, não considera as classificações ou notas atribuídas entre os pares. Para esta docente, a avaliação do desempenho do estudante nessas atividades é um fator que pode impactar significativamente nos resultados da aprendizagem de ambos (avaliador e avaliado), e sem ela, possivelmente o empenho dos estudantes seria reduzido. Prof2 desenvolve OPA em um ciclo de atividades realizado em 4 etapas: (a) Todos os estudantes devem elaborar um texto de análise crítica sobre um mesmo artigo científico escolhido pela docente, relacionado ao tema que está sendo discutido nas aulas. (b) Em 
VII Congresso Brasileiro de Informática na Educação (CBIE 2018)

Anais do XXIV Workshop de Informática na Escola (WIE 2018)

seguida, o par-avaliador faz, segundo uma grelha FRISCO [Ennis 1996; Nelson e Shum 2009], uma série de indicações num documento que é partilhado com todos os demais e discutido na sala de aula. (c) O par-avaliado recebe as revisões do seu par e com base nelas, melhora seu trabalho e apresenta contra-argumentações ao seu par; (d) O paravaliador faz nova revisão do trabalho do seu par e apresenta seus últimos comentários. Após a conclusão desse ciclo cada estudante apresenta seu trabalho final, desenvolvido em colaboração entre pares. A avaliação do desempenho do trabalho de cada estudante é feita pela docente, sendo ela composta por 20 valores os quais são distribuídos em subtrabalhos desenvolvidos em diversos momentos do ciclo. Este mesmo ciclo é repetido 3 vezes por semestre.

Prof3 por sua vez, considera a classificação atribuída pelos pares-avaliadores complementada com a sua avaliação, sendo que, a avaliação sobre um artigo de revisão bibliográfica é a mais utilizada.

Pelo exposto, há uma variação nos tipos de procedimentos de OPA desenvolvidos pelas(os) docentes entrevistados: a avaliação do desempenho do grupoavaliador e do grupo-avaliado, atribuída pela(o) docente (prof1); avaliação do desempenho, atribuída pela(o) docente, tanto do par-avaliado quanto do par-avaliador (prof2); a classificação atribuída pelos pares-avaliadores complementada com a avaliação da(o) docente (prof3). Na literatura há indicações de que métodos de avaliação de OPA em que são consideradas as classificações dos estudantes aos seus paresavaliados, são propícias por ajudar a resolver problemas relacionados com o volume de trabalho dos docentes e de turmas com grande número de estudantes; e por permitir poupar tempo de classificação [Fagerholm e Vihavainen 2013].

No inquérito realizado com os estudantes, buscamos conferir suas compreenções ou pontos de vista acerca das diretivas de avaliação utilizadas pelas(os) docentes na realização de OPA, conforme o Gráfico 1:

\section{Gráfico 1 - Ponto de vista dos estudantes $(n=50)$ relacionadas ao método de avaliação}

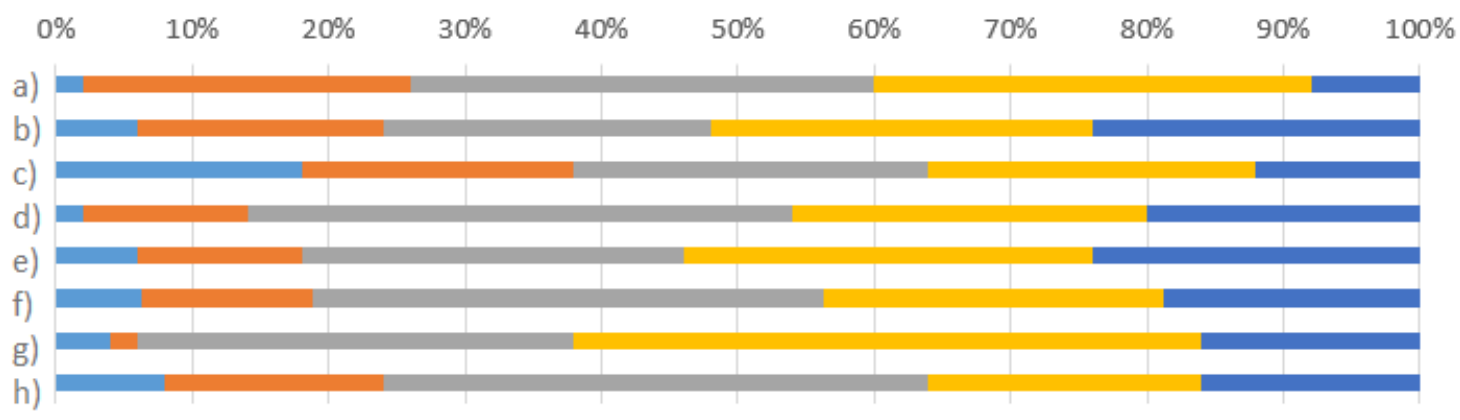

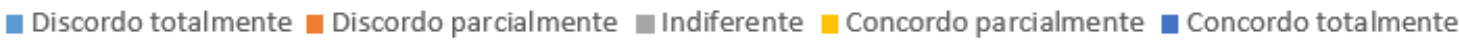

No Gráfico 1, as questões são as seguintes: a) Considero difícil avaliar o(s) meu(s) par(es) atribuindo notas quantitativas aos seus trabalhos; b) Quando faço a avaliação pelos pares, prefiro emitir feedback oral do que escrito; c) Nas avaliações pelos pares, considero justo receber a mesma nota dos outros estudantes do meu grupo; d) Os meus pares avaliam-me de forma justa; e) Avaliar meus colegas de forma anônima, permite-me fazê-lo de forma mais justa; f) Sinto-me mais confortável ao fazer avaliação pelos pares de forma anônima; g) Contra argumentar a avaliação que recebo do(s) meu(s) par(es) potencia a minha aprendizagem; h) Quando a avaliação pelos pares é contabilizada na minha nota final, comprometo-me mais.

A avaliação pelos pares é uma estratégia em que os estudantes julgam o desempenho de um par quantitativamente, fornecendo uma nota com pontuações e/ou 
VII Congresso Brasileiro de Informática na Educação (CBIE 2018)

Anais do XXIV Workshop de Informática na Escola (WIE 2018)

qualitativamente, fornecendo um feedback escrito ou oral. Neste sentido, de acordo com o exposto no Gráfico 1, os resultados mostram que há um certo equilíbrio na percentagem de estudantes que consideram este método difícil ou fácil e, além disso, os estudantes inqueridos revelaram maior preferência pelo feedback oral [Santos Rosa, Coutinho e Flores 2017].

De acordo com os dados citados acima, relembrando que os dados foram organizados em uma escala Likert, sendo de 01 (discordo totalmente) à 05 (concordo totalmente), a atribuição de notas quantitativas aos seus pares é considerada de difícil aplicação por $24 \%$ dos estudantes; $34 \%$ manifestaram que não concordam nem discordam; e $32 \%$ concordam e $8 \%$ concordam fortemente. Estes indicadores nos remetem a considerar que há uma falta de consenso, e com isso, caberá ao docente analisar o contexto em que a OPA está inserida para adotar esta estratégia. Outro ponto a se destacar é que a maioria dos estudantes preferem emitir feedback oral ao invés de escrito ( $28 \%$ concordam e $24 \%$ concordam totalmente); $24 \%$ não concordam nem discordam; $18 \%$ discordam e $6 \%$ discordam totalmente.

Quando questionados sobre se consideram justo receber a mesma nota dos outros estudantes do grupo nas avaliações pelos pares: 24\% concordam e $12 \%$ concordam totalmente; $26 \%$ não concordam nem discordam e; $20 \%$ discordam e $18 \%$ discordam totalmente e; a maioria (40\%) não concordam nem discordam que seus pares os avaliam de forma justa; $26 \%$ concordam e $20 \%$ concorda totalmente; já $12 \%$ discordam e $2 \%$ discordam totalmente.

Quando questionados se avaliar seus colegas de forma anônima, permite-lhes fazê-lo de forma mais justa, $30 \%$ concordam e $24 \%$ concordam totalmente; 28 não concordam e nem discordam; e 12\% discordam e $6 \%$ discordam totalmente. Sobre a preservação do anonimato na OPA, 36\% não concordam nem discordam; $24 \%$ concordam e $8 \%$ concordam totalmente; $12 \%$ discordam e $6 \%$ discordam totalmente. Os pontos de vista dos estudantes relacionadas a questão de justiça e da manutenção do anominato ou não, tem sido alvo de discussão em pesquisas recentes sobre avaliação pelos pares, a qual tem sido incentivada por possibilitar o anonimato da autoria de forma mais eficiente [Wen e Tsai 2006] ao permitir que os estudantes expressem livremente as suas ideias sobre o trabalho dos seus pares e por reduzir restrições relacionadas ao horário e localização [Issa 2012]; por favorecer uma avaliação mais honesta e justa [Jimenez-Romero, Johnson e Castro 2013; Bouchoucha e Wozniak 2010];

A maioria dos estudantes afirma que contra argumentar a avaliação recebida por seus pares potencializa sua aprendizagem (46\% concordam e $16 \%$ concordam totalmente). Já 32\% não concordam nem discordam; $2 \%$ discordam e $4 \%$ discordam totalmente. De modo geral, avaliações pelos pares no ensino superior têm demonstrado ser uma estratégia pedagógica propícia para promover a aprendizagem [Falchikov e Goldfinch 2000], porque pode aumentar competências de trabalho colaborativo essenciais para o desenvolvimento profissional [Bryan 2006] e dar maior sentido de responsabilidade e de motivação ao estudante [Boud e Falchikov 2005]. Estudantes que dela participam, emitem maiores níveis de compreensão dos assuntos abordados e de reflexão sobre sua própria compreensão, fato que resulta em níveis de aprendizagem profundos ao invés de aprendizagens superficiais.

Para finalizar esta categoria, inquirimos se quando a avaliação pelos pares é contabilizada na nota final do estudante ele se compromete mais. Como resultado, a maioria $(40 \%)$ dos estudantes afirmaram não discordar nem concordar com esta 
VII Congresso Brasileiro de Informática na Educação (CBIE 2018)

Anais do XXIV Workshop de Informática na Escola (WIE 2018)

afirmação; $20 \%$ concordam e $16 \%$ concordam totalmente, restando apenas $16 \%$ que discordam e $8 \%$ que discordam totalmente.

Pelo acima exposto, podemos aferir que as diferentes compreensões dos estudantes acerca dos procedimentos da avaliação podem conduzir os docentes a reflexões sobre qual melhor se adequa às suas práticas de OPA.

\subsection{Tecnologias Digitais utilizadas em contextos de OPA}

Uma OPA, geralmente gera um produto: um texto em que os estudantes respondem à questão problema anunciada pelo docente (prof1) ou um texto de análise crítica sobre um artigo científico (prof2). Durante o processo de produção estudantes e docentes fazem uso de Tecnologias Digitais tanto para a elaboração do produto final avaliado, quanto para a interação, colaboração e avaliação. Os recursos utilizados para desenvolver OPA pelos docentes entrevistados são: Google drive (prof1, prof2, prof3) e o e-mail (prof1, prof3) utilizado após a aula para envio de documento, quando ocorriam problemas de conexão durante a aula. O Excel, o Word, o Facebook, o Skype e o Dropbox foram citados por prof4, para que os estudantes e o docente possam elaborar, atualizar e acompanhar conjuntamente: atas das reuniões que desenvolvem, calendarização das atividades, registros das avaliações. Segundo prof4 "[...] toda a gestão da informação é feita nestas ferramentas".

Com relação ao uso das Tecnologias Digitais não constatamos dificuldades técnicas referente ao seus usos instrumentais relevantes, nem dos docentes nem dos estudantes. Entretanto, tomando como exemplo o Google drive, mesmo se tratando de uma ferramenta com facilidade de uso, é possível que num primeiro momento, seja necessário realizar uma aula-teste para apresentar exemplos práticos de como utilizá-lo. Do ponto de vista da gestão dos resultados, nenhum dos recursos supracitados possuem ferramentas prontas para gerenciar os relacionamentos entre os dados gerados nas OPA. O Google formulário, por exemplo, gera um relatório em uma planilha de cálculo que pode, a partir de bons conhecimentos em fórmulas avançadas, ser utilizado para gerenciar os dados coletados pelo docente em uma OPA. Mas, esta solução não atende a maioria dos docentes que não possuem conhecimentos avançados para isso. Da mesma forma, os demais recursos supracitados, por não serem desenvolvidos para atender necessidades específicas de OPA, tornam-a inviável quando aplicada para grandes demandas (muitos alunos ou de uso frequente). Neste sentido, as pesquisas em Educação e em Desenvolvimento tecnológico devem avançar com contribuições específicas para OPA.

No gráfico 2, apresentamos as respostas dos estudantes às questões relacionadas ao uso de Tecnologias Digitais na OPA:

\section{Gráfico 2 - Ponto de vista dos estudantes $(n=50)$ sobre o uso de TECNOLOGIAS}

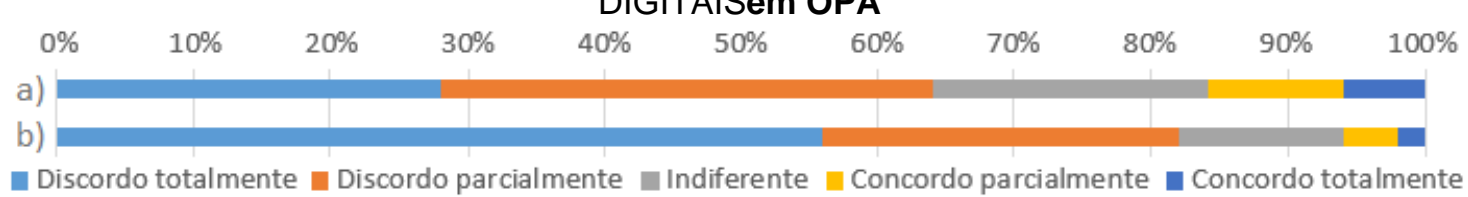

Pelo exposto no Gráfico 2 - cujas questões foram: a) Tenho dificuldades em utilizar as Tecnologias Digitais para fazer a avaliação pelos pares; b) As Tecnologias Digitais que utilizo na avaliação pelos pares são desadequadas porque não possibilitam meu anonimato, - a maioria dos estudantes não possuem dificuldades em utilizar 
VII Congresso Brasileiro de Informática na Educação (CBIE 2018)

Anais do XXIV Workshop de Informática na Escola (WIE 2018)

Tecnologias Digitaispara desenvolver suas respectivas OPA (56\% discordam totalmente e $26 \%$ discordam que há dificuldades) e, além disso, discordam $(56 \% ; 26 \%)$ que as Tecnologias Digitais que utilizam são desadequadas.

Na literatura, constatamos que o uso das Tecnologias Digitais, por meio das quais busca-se desenvolver OPA, encontram-se em dois patamares, por um lado são exploradas as já existentes e desenvolvidas para finalidades diversificadas, como por exemplo: google docs, wiki, editores de textos, os ambientes virtuais de aprendizagem, sistema de resposta ao aluno [Bouchoucha e Wozniak 2010; Chang et al. 2011; Chen et al. 2009; Dominguez et al. 2013]. Por outro lado, com o aumento das pesquisas e das práticas de OPA, têm-se desenvolvido ferramentas para atender necessidades específicas como, por exemplo, o "PASS" proposto por Al-Smadi, Guetl e Kappe (2009), o "Guess it! " proposto por Berns et al. (2012), cuja finalidade é explorar a inteligência coletiva na aprendizagem de línguas utilizando dispositivos de comunicação móvel e o Peer Grade [PeerGrade 2018], um sistema online de criação de OPA utilizando rubricas.

Em nossas pesquisas sobre OPA [Santos Rosa, Coutinho e Flores 2017], temos considerado que as Tecnologias Digitais, podem ajudar as(os) docentes construírem estratégias de avaliação para fazer julgamentos válidos do progresso da aprendizagem do aluno, facilitar a troca de feedback e apoiar a aprendizagem colaborativa. Tais recursos são utilizados para proporcionar: mais flexibilidade relacionada com os horários e locais de estudo e oferecer oportunidades para o estudante gerenciar, rever e avaliar sua própria aprendizagem; possibilitar a manutenção de registros das diferentes fases de um trabalho/atividade; dispor de ferramentas de monitoramento das alterações feitas após o feedback do(s) par(es), as quais podem ser mais facilmente identificadas, bem como, fazer uso de sistemas computacionais como os tutores inteligentes que permitem ao estudante obter feedback dinâmico durante o ato da produção de uma resposta.

Como sugestões para melhorar a eficácia e utilidade da OPA, os estudantes apontaram: a necessidade de um maior número de avaliações; a definição de critérios mais específicos e não ambíguos; a realização de avaliações individuais ao invés de em grupo para que se valorize cada indivíduo do grupo. Segundo um dos estudantes, para evitar injustiças essas avaliações individuais podem evitar conflitos gerados entre os elementos de um mesmo grupo: "se metade do grupo se desentender com outra metade pode prejudicar aqueles que realmente contribuíram para o trabalho e assim tornar-se injusto" (aluno). Outra componente que pode contribuir contra injustiças e conflitos consiste no diálogo entre os estudantes: "dialogar sobre os valores dados a cada parâmetro de forma a perceber se a avaliação foi feita com consciência e o porquê de certas notas" (aluno). Além disso, é necessário "melhorar os critérios de avaliação, tornando-os mais especificos" (aluno). Por fim, consideram importante realizar avaliações contínuas: "não esperar até os pontos de controle de um projeto estar concluídos para se efetuar a avaliação pelos pares" (aluno) e; possibilitar uma preparação prévia para as avaliações classificatórias: "os resultados dessa avaliação devem servir para incentivar os menos empenhados e dar-lhes a oportunidade de melhorar antes dos momentos de avaliação" (aluno).

Nas práticas de OPA, alguns dos princípios de avaliação propostos com o uso dessas tecnologias podem ser utilizados para contribuir com um ponto de equilíbrio entre a avaliação enquanto um instrumento de medição do desempenho do estudante (avaliação da aprendizagem) para uma avaliação que o envolva ativamente nos processos de avaliação da sua própria aprendizagem (avaliação para a aprendizagem). 
VII Congresso Brasileiro de Informática na Educação (CBIE 2018)

Anais do XXIV Workshop de Informática na Escola (WIE 2018)

\section{Considerações finais}

Ao longo deste artigo apresentamos a partir das práticas docentes e dos pontos de vista dos estudantes, como a OPA tem sido desenvolvida no ensino superior. Identificamos os procedimentos aplicados em OPA e as Tecnologias Digitais utilizadas e, por fim, relacionamos as compreensões de estudantes com elementos das práticas de OPA elencadas por docentes alinhadas a pesquisas afins. Os pontos de vista dos estudantes e dos docentes conduzem a reflexões sobre elementos da OPA que podem inviabilizar sua utilização, bem como, causar conflitos, injustiças e consequentemente prejuízos no processo de ensino e aprendizagem. Por outro lado, constatamos evidências de que, se bem planejada e conduzida, a OPA pode contribuir com a concepção de feedback, alvos dos docentes participantes da presente pesquisa.

Salientamos que os resultados desta pesquisa servirão de base para o desenvolvimento de um software específico para OPA no qual serão consideradas suas particularidades diante das possibilidade tecnológicas atuais, com elementos computacionais de aprendizagem de máquina, por exemplo, tecnicas de learning analytic e tutores inteligentes. Assim, sendo a avaliação um elemento crucial no processo de ensino e aprendizagem e pelo fato de que as possibilidades tecnológicas atuais possibilitam, mas não cobrem todas as necessidades específicas dos diversos métodos de ApA, consideramos que esta é uma área que exige significativo investimento colaborativo e, neste sentido, ferramentas online para apoiar a nova geração de estudantes em processos de avaliação, será um foco para pesquisas e desenvolvimento futuros.

\section{Referencias}

Al-Smadi, M.; Guetl, C.; Kappe, F. Pass. (2009). Peer-ASSessment Approach for Modern Learning Settings. M. Spaniol et al. (Eds.): ICWL 2009, LNCS 5686, 44-47.

Assessment Reform Group - ARG (1999). Assement for learning: Beyond the Black

Box. University of Cambridge School of Education: 1999. Disponível em: <http://www.nuffieldfoundation.org/sites/default/files/files/beyond_blackbox.pdf > Acesso em 16/04/2018.

Berns, A.; Palomo-Duarte, M.; Dodero, J.M.; Cejas, A. (2012). Guess it! Using gamicated apps to support students foreign language learning by organic community-driven peer-assessment. Open Learning and Teaching in Educational Communities, Austria, v. 8719, p. 482-485.

Bouchoucha, S.; Wozniak, H. (2010). Is PA of asynchronous group discussions fostering skills relevant to our future graduates? Proceedings ascilite Sydney.

Boud, D. and Falchikov, N. (2005) 'Redesigning assessment for learning beyond higher education', in Research and Development in Higher Education 28, A. Brew and C. Asmar (eds), 34-41.

Bryan, C. (2006). Developing group learning through assessment. In C. Bryan e K. Clegg (Eds.), Innovative assessment in higher education. Oxon: Routledge.

Caseiro, C.C.F.; Gebran, R.A. Avaliação Formativa: Concepção, Práticas e Dificuldades. Nuances: estudos sobre Educação. Presidente Prudente, SP, ano XIV, v. 15, n. 16, p. 141-161, jan./dez. 2008. 
VII Congresso Brasileiro de Informática na Educação (CBIE 2018)

Anais do XXIV Workshop de Informática na Escola (WIE 2018)

Chang, S-H.; Chen, M-L.; Kuo,Y-K.; Shen, Y-C. (2011). A Simulation-Based LED Design Project in Photonics Instruction Based on Industry-University Collaboration. IEEE Transactions on Education, Vol. 54, no. 4.

Chen, N-S.; Wei, C-W.; Wua, W-T.; Uden, L. (2009). Effects of high-level prompts and PA on online learners' reflection levels. Computers e Education 52 (2009) 283-291.

Dominguez, C., Nascimento, M., Maia, A., Pedrosa, D., Cruz, G. (2013). Insights on web-based peer review: A case study with energy engineering students. 1st International Conference of the Portuguese Society for Engineering Education, CISPEE.

Ennis, R. H. (1996). Critical thinking. Upper Saddle River, N. J.: Prentice Hall.

Eugenia M. W. N. G. (2014). Using a mixed research method to evaluate the effectiveness of formative assessment in supporting student teachers' wiki authoring. Computers e Education 73, 141-148.

Falchikov, N.; Goldfinch, J. (2000). Student peer assessment in higher education: a meta-analysis comparing peer and teacher marks. Review of Educational Research, 70(3), 287-322.

Fagerholm, F.; Vihavainen, A. (2013). Peer Assessment in Experiential Learning: Assessing Tacit and Explicit Skills in Agile Software Engineering Capstone Projects.

Grez, L.; Valcke, M. (2013). Student Response System and How to Make Engineering Students Learn Oral Presentation Skill. International Journal of Engineering Education Vol. 29, No. 4, 940-947.

Issa, T. (2012). Promoting learning skills through teamwork assessment and self/peer evaluation in higher education. IADIS International Conference on Cognition and Exploratory Learning in Digital Age. CELDA.

Jimenez-Romero, C. , Johnson, J., Castro, R. D. (2013). Máquina e sistemas peeravaliação inteligentes sociais para avaliar populações estudantis grandes na educação massivo online aberta. Anais da Conferência Europeia sobre e-Learning, ECEL The future of global learning engineering education.

Nicol, D. (2011). Developing students' ability to construct feedback. Retrieved from http://www.enhancementthemes.ac.uk/docs/publications/developing-students-abilityto-construct-feedback.pdf?sfvrsn=30.

Peer Grade. PeerGrade. (2018). Disponível em www.peergrade.io. Acessado em 28 de maio de 2018.

Santos Rosa, S. Coutinho, C.P., Flores, M.A (2017). Online Peer Assessment no ensino superior: uma revisão sistemática da literatura em práticas educacionais. Avaliação, Campinas; Sorocaba, SP, v. 22, n. 1, p. 55-83.

Sluijsmans, D. M. A; Straetmans, J. J. M.; Van Merriënboer , J. G. (2008). Integrating authentic assessment with competence-based learning in vocational education: the protocol portfolio scoring. Journal of Vocational Education and Training. Vol. 60, $2^{\mathrm{a}}$ edição. p. 159-172.

Wen, M. L; Tsai, C.C. (2006). University Students' Perceptions of and Attitudes Toward (Online) PA. Higher Education. Volume 51, Issue 1, 27-44. 